\title{
SHAPE OPTIMIZATION FOR DIRICHLET PROBLEMS: RELAXED SOLUTIONS AND OPTIMALITY CONDITIONS
}

\author{
GIUSEPPE BUTTAZZO AND GIANNI DAL MASO
}

\begin{abstract}
We study a problem of shape optimal design for an elliptic equation with Dirichlet boundary condition. We introduce a relaxed formulation of the problem which always admits a solution, and we find necessary conditions for optimality both for the relaxed and the original problem.
\end{abstract}

Let $\Omega$ be a bounded open subset of $\mathbf{R}^{n}(n \geq 2)$, let $f \in L^{2}(\Omega)$, and let $g: \Omega \times \mathbf{R} \rightarrow \mathbf{R}$ be a Carathéodory function (i.e. $g(x, s)$ measurable in $x$ and continuous in $s$ ) such that

$$
|g(x, s)| \leq a_{0}(x)+b_{0}|s|^{2} \quad \forall(x, s) \in \Omega \times \mathbf{R},
$$

for suitable $a_{0} \in L^{1}(\Omega)$ and $b_{0} \in \mathbf{R}$. We consider the following optimal design problem:

$$
\min _{A \in \mathscr{A}(\Omega)} \int_{\Omega} g\left(x, u_{A}(x)\right) d x,
$$

where $\mathscr{A}(\Omega)$ is the family of all open subsets of $\Omega$, and $u_{A}$ is the solution of the Dirichlet problem

$$
-\Delta u_{A}=f \text { in } A, \quad u_{A} \in H_{0}^{1}(A),
$$

extended by 0 in $\Omega \backslash A$.

It is well known that, in general, the minimum problem (1) has no solution (see for instance Example 2). The reason is that, although the solutions $u_{A_{h}}$ of (2) corresponding to a minimizing sequence $\left(A_{h}\right)$ of (1) always admit a limit point $u$ in the weak (not necessarily in the strong) topology of $H_{0}^{1}(\Omega)$, we can not find, in general, an open subset $A$ of $\Omega$ such that $u=u_{A}$. On the contrary, it can be proved (see [4]) that the limit function $u$ is the solution of a relaxed Dirichlet problem of the form

$$
-\Delta u+\mu u=f \text { in } \Omega, \quad u \in H_{0}^{1}(\Omega) \cap L^{2}(\Omega ; \mu),
$$

Received by the editors May 17, 1989 and, in revised form, November, 1989. 1980 Mathematics Subject Classification (1985 Revision). Primary 49A50. 
for a suitable nonnegative measure $\mu$ which vanishes on all sets of (harmonic) capacity 0 , but may take the value $+\infty$ on some subsets of $\Omega$. Following [3], we shall denote by $\mathscr{M}_{0}(\Omega)$ the class of all measures with the properties considered above.

The precise meaning of equation (3) is the following:

$$
\int_{\Omega} D u D \varphi d x+\int_{\Omega} u \varphi d \mu=\int_{\Omega} f \varphi d x
$$

for every $\forall \varphi \in H_{0}^{1}(\Omega) \cap L^{2}(\Omega ; \mu)$, where the pointwise value of an $H^{1}$ function is defined as usual up to sets of capacity 0 .

If $S$ is a Borel subset of $\Omega$, the measure $\infty_{S}$ defined by

$$
\infty_{S}(B)= \begin{cases}0 & \text { if } B \cap S \text { has capacity } 0 \\ +\infty & \text { otherwise }\end{cases}
$$

belongs to $\mathscr{M}_{0}(\Omega)$. Note that if $S$ is closed in $\Omega$, then problem (3) reduces to problem (2) with $A=\Omega \backslash S$ and $\mu=\infty_{S}$. The relaxed formulation of the optimization problem (1) is then:

$$
\min _{\mu \in \mathscr{M}_{0}(\Omega)} \int_{\Omega} g\left(x, u_{\mu}(x)\right) d x,
$$

where $u_{\mu}$ is the unique solution of the relaxed Dirichlet problem (3) in the sense given by (4).

The following theorem follows easily from the compactness and density results for relaxed Dirichlet problems proved in [1] (Theorem 2.38) and [4] (Theorem 4.16).

Theorem 1. Problem (6) admits a solution, and

$$
\min _{\mu \in \mathscr{M}_{0}(\Omega)} \int_{\Omega} g\left(x, u_{\mu}(x)\right) d x=\inf _{A \in \mathscr{A}(\Omega)} \int_{\Omega} g\left(x, u_{A}(x)\right) d x .
$$

We now give an example where problem (1) has no solution.

Example 2. Assume that $f(x)>0$ a.e. in $\Omega$, let $w$ be the solution of

$$
-\Delta w=f \text { in } \Omega, \quad w \in H_{0}^{1}(\Omega),
$$

and let $g(x, s)=|s-c w(x)|^{2}$, with $0<c<1$. Then the relaxed problem (6) attains its minimum value 0 at the measure $\mu$ defined by

$$
\mu(B)=\frac{1-c}{c} \int_{B} \frac{f}{w} d x
$$

which corresponds to $u_{\mu}=c w$. On the other hand, it is clear from (2) and (8) that there are no domains $A$ for which $g\left(x, u_{A}(x)\right)=$ 
0 a.e. in $\Omega$. By (7) this implies that the original problem (1) has no solution.

Our goal is to find optimality conditions for the solutions of problem (6). We recall that the fine topology on $\Omega$ is the weakest topology on $\Omega$ for which all superharmonic functions are continuous. For a systematic study of properties of the fine topology we refer to Doob [5], Part 1, Chapter XI. Let $\mu$ be a minimum point of (6) and let $u=u_{\mu}$. By $A=A(\mu)$ we denote the set of all $x \in \Omega$ having a fine neighborhood $V$ such that $\mu(V)<+\infty$, and by $\mu_{A}$ the restriction of $\mu$ to $A$; it is clear that $A$ is finely open in $\Omega$. By $\partial^{*} A$ and $c l^{*} A$ we denote the fine boundary and the fine closure of $A$ in $\Omega$.

Proposition 3. There exist a Radon measure $\nu \in \mathscr{M}_{0}(\Omega)$ carried by $\partial^{*} A$, and a continuous linear map $T: L^{2}(\Omega) \rightarrow L^{2}\left(\partial^{*} A, \nu\right)$ such that, if $h \in L^{2}(\Omega)$ and $w \in H_{0}^{1}(\Omega) \cap L^{2}(\Omega ; \mu)$ is a solution of

$$
-\Delta w+\mu w=h \quad \text { in } \Omega
$$

in the sense given by (4), then

$$
\int_{A} D w D \varphi d x+\int_{\partial^{*} A} T(h) \varphi d \nu+\int_{A} w \varphi d \mu_{A}=\int_{c l^{*} A} h \varphi d x
$$

for every $\varphi \in H_{0}^{1}(\Omega)$.

If $A$ is an open set with a smooth boundary and $\mu_{A}(B)=$ $\int_{B \cap A} \vartheta d x$ with $\vartheta \in L^{\infty}(\Omega)$, an integration by parts leads to the form

$$
\nu(B)=-\int_{B \cap \partial A} \frac{\partial W}{\partial n} d \sigma, \quad T(h)=\frac{\partial w / \partial n}{\partial W / \partial n}
$$

where $\sigma$ denotes the surface measure on the (Euclidean) boundary $\partial A$ of $A, n$ is the outer unit normal to $A$, and $W$ is the solution of the Dirichlet problem

$$
-\Delta W=1 \text { in } A, \quad W \in H_{0}^{1}(A) .
$$

In addition to the previous hypotheses, we assume now that $g(x, s)$ is continuously differentiable with respect to $s$ and that

$$
\left|g_{s}(x, s)\right| \leq a_{1}(x)+b_{1}|s| \quad \forall(x, s) \in \Omega \times \mathbf{R}
$$

for suitable $a_{1} \in L^{1}(\Omega)$ and $b_{1} \in \mathbf{R}$.

In order to give our optimality conditions, we introduce the adjoint equation

$$
-\Delta v+\mu v=g_{s}, \quad v \in H_{0}^{1}(\Omega) \cap L^{2}(\Omega ; \mu),
$$


where $g_{s}$ denotes the function $g_{s}(x, u(x))$. We denote by $v$ the solution of (10) in the sense given by (4), with $f$ replaced by $g_{s}$, and we set

$$
\alpha=T(f), \quad \beta=T\left(g_{s}\right) .
$$

Our main result is the following theorem.

Theorem 4. Let $\mu=\infty_{S}+\mu_{A}$ be a solution of problem (6), let $u=u_{\mu}$ be the corresponding solution of (3), and let $v$ be the solution of the adjoint equation (10). If $A=A(\mu)$, then $u=v=0$ on $\Omega \backslash A$ ( up to a set of capacity 0 ), and

(a) $u v \leq 0$ a.e. on $A$,

(b) $\alpha \beta \geq 0 \quad \nu$-a.e. on $\partial^{*} A$,

(c) $f(x) g_{s}(x, 0) \geq 0$ a.e. on $\Omega \backslash c l^{*} A$,

(d) $u v=0 \quad \mu_{A}$-a.e. on $A$,

where $\alpha$ and $\beta$ are given by (11).

Suppose now that there exists an optimal domain $A$ for the original problem (1), and that $A$ has a smooth boundary. By (7) the measure $\mu=\infty_{S}$ defined by (5) with $S=\Omega \backslash A$ is a minimum point of the relaxed problem (6). Taking (9) into account, the optimality conditions of Theorem 4 become:

$\left(\mathrm{a}^{\prime}\right) \quad u v \leq 0$ a.e. on $A$,

(b') $\frac{\partial u}{\partial n} \frac{\partial v}{\partial n} \geq 0 \quad \sigma$-a.e. on $\Omega \cap \partial A$,

(c') $f(x) g_{s}(x, 0) \geq 0$ a.e. on $\Omega \backslash A$,

while condition (d) is trivial because $\mu_{A}=0$. From $\left(\mathbf{a}^{\prime}\right)$ and $\left(\mathbf{b}^{\prime}\right)$ we obtain

$$
\frac{\partial u}{\partial n} \frac{\partial v}{\partial n}=0 \sigma \text {-a.e. on } \Omega \cap \partial A \text {. }
$$

The last condition is already known in shape optimization (see for instance $[2,9,10,13,15])$, while conditions $\left(a^{\prime}\right)$ and $\left(c^{\prime}\right)$ seem to be new. Similar relaxed formulations for different classes of optimal design problems (with Neumann or other boundary conditions) have been considered by Murat and Tartar in $[8,11$, $12,14]$, and by Kohn, Strang, and Vogelius in [6, 7].

\section{REFERENCES}

1. H. Attouch, Variational convergence for functions and operators, Pitman, London, 1984.

2. J. Cea, Problems of shape optimal design, Proceedings of Optimization of Distributed Parameter Structures, Iowa City, 1980, Sijthoff and Noordhoff, Rockville, 1981, pp. 1005-1048. 
3. G. Dal Maso, $\Gamma$-convergence and $\mu$-capacities, Ann. Scuola Norm. Sup. Pisa Cl. Sci. (4) 14 (1987), 423-464.

4. G. Dal Maso and U. Mosco, Wiener's criterion and $\Gamma$-convergence, Appl. Math. Optim. 15 (1987), 15-63.

5. J. L. Doob, Classical potential theory and its probabilistic counterpart, Springer-Verlag, Berlin, 1984.

6. R. V. Kohn and G. Strang, Optimal design and relaxation of variational problems, I, II, III, Comm. Pure Appl. Math. 39 (1986), 113-137, 139-182, 353-377.

7. R. V. Kohn and M. Vogelius, Relaxation of a variational method for impedance computed tomography, Comm. Pure Appl. Math. 40 (1987), 745-777.

8. F. Murat, Control in coefficients, Encyclopedia of Systems and Control, Pergamon Press, Elmsford, N.Y., 1983, pp. 808-812.

9. F. Murat and J. Simon, Etude de problemes d'optimal design, Proceedings of Optimization Techniques. Modelling and Optimization in the Service of Man, Part 2, Nice 1975, Lecture Notes in Comput. Sci. 41 (1976), 54-62.

10. F. Murat and J. Simon, Sur le contrôle par un domaine géometrique, Univ. Paris VI, preprint 76015, 1976.

11. F. Murat and L. Tartar, Calcul des variations et homogénéisation, Proceedings of Les Méthodes de l'homogénéisation: Théorie et applications en physique, Ecole d'Eté d'Analyse Numérique C.E.A.-E.D.F.-INRIA, Bréausans-Nappe 1983, Collect. Dir. Études Rech. Elec. France 57 (1985), 319369.

12. F. Murat and L. Tartar, Optimality conditions and homogenization, Proceedings of Nonlinear Variational Problems, Isola d'Elba 1983, Res. Notes Math. 127 (1985), 1-8.

13. O. Pironneau, Optimal shape design for elliptic systems, Springer-Verlag, Berlin, 1984.

14. L. Tartar, Problèmes de contrôle des coefficients dans des équations aux dérivées partielles, Control Theory, Numerical Methods and Computer Systems Modelling, Lecture Notes in Econom. and Math. Systems 107 (1975), 420-426.

15. J. P. Zolesio, The material derivative (or speed) method for shape optimization, Proceedings of Optimization of Distributed Parameter Structures, Iowa City, 1980, Sijthoff and Noordhoff, Rockville, 1981, pp. 10891151 .

Dipartimento di Matematica, Via Machiavelli, 3544100 Ferrara, Italy

S.I.S.S.A., Strada Costiera, 1134014 Trieste, Italy 
